\title{
Nasopharyngeal carcinoma with esophageal metastasis presenting with progressive dysphagia
}

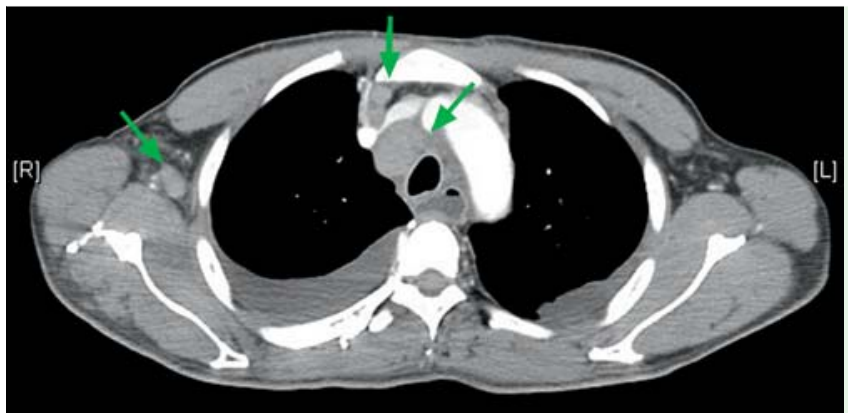

Fig. 1 Contrastenhanced thoracic computed tomography (CT) scan showing metastatic spread to axillary, prevascular, paratracheal, precarinal, subcarinal, paraesophageal, perigastric, para-aortic, and intercavo-aortic lymph nodes.
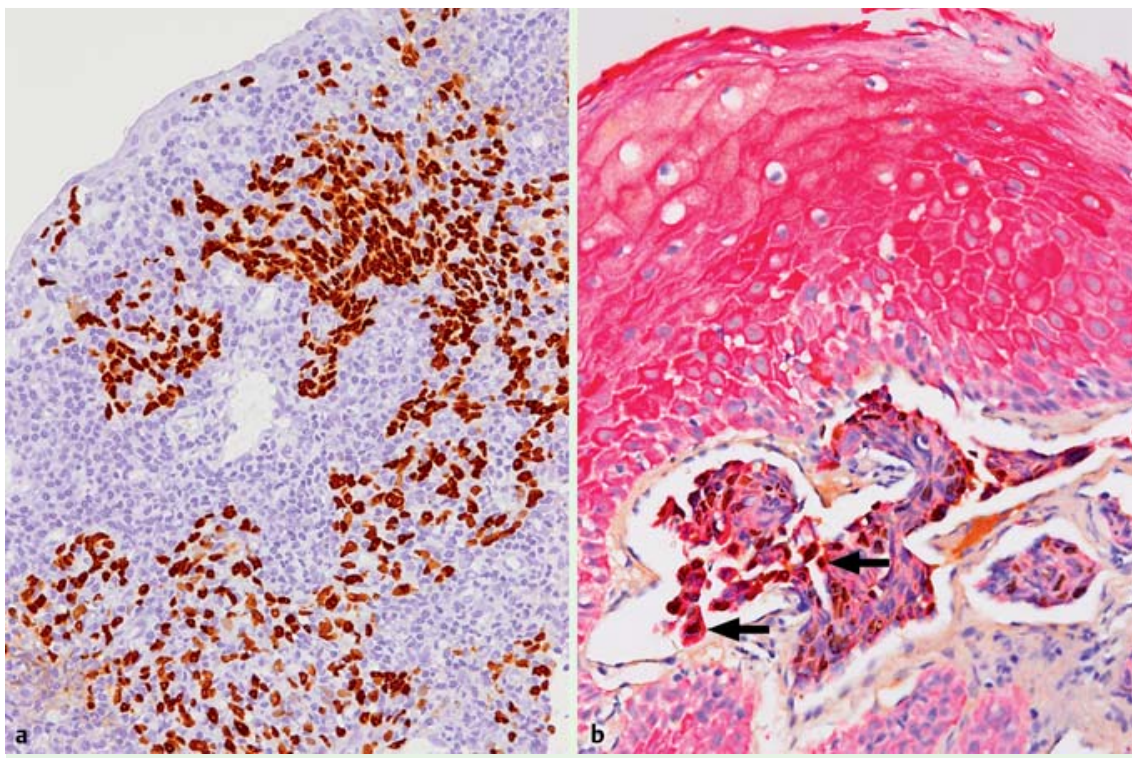

Fig. 3 Histology of the esophageal biopsy specimen. a In situ hybridization for Epstein-Barr virus (EBV)-encoded RNA-1 (EBER-1) with DAB (3,3'-diaminobenzidine) chromogen showing brown positive nuclear staining (original magnification $\times 200$ ). b Immunostaining for high molecular weight cytokeratin with AEC (3-amino-9-ethylcarbazole) chromogen showing red positive cytoplasmic staining (original magnification $\times 200$ ). The arrow indicates cells that are showing dual staining.

A 49-year-old man attended our department complaining of progressive difficulty in swallowing over a period of 2 weeks. He reported no other discomfort. His medical history revealed that he had been diagnosed with nasopharyngeal carcinoma (cT3N3bM0; WHO Type III) in September 2008 and had undergone chemotherapy and radiotherapy concurrently. A complete response was achieved initially, but regional recurrence in the right side of his neck required him to undergo neck dissection in June 2010. Progressive dysphagia brought him to our department 4 months later. Imaging studies of the head and neck region produced negative findings, but an esophagogram revealed narrowing in the middle third of his esophagus.

Contrast-enhanced thoracic computed tomography (CT) demonstrated multiple nodal metastases in the thoracic cavity and enlargement of the retrocardiac segment of the esophagus ( $\bullet$ Fig. 1 ). Esophagoscopy demonstrated a protruding mass located $30-35 \mathrm{~cm}$ from the upper incisors ( $\bullet$ Fig.2). Biopsy showed a poorly differentiated carcinoma; metastasis from the nasopharyngeal carcinoma was suspected. In addition, in situ hybridization showed that the neoplastic epithelial cells were positive for both Epstein-Barr virus (EBV)-encoded RNA-1 (EBER-1; $\bullet$ Fig.3a) and high molecular weight cytokeratin

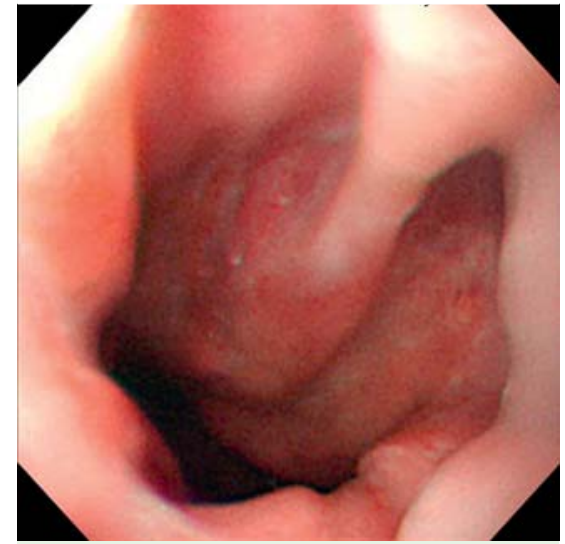

Fig.2 Esophagoscopy showing lumen narrowing and a protruding mass between 30 and $35 \mathrm{~cm}$ from the upper incisors.

$(\bullet$ Fig. 3b). These findings were consistent with metastatic nasopharyngeal carcinoma, as in situ hybridization results from a previous nasopharyngeal specimen were also positive for EBER-1 in neoplastic epithelial cells. The patient underwent palliative chemotherapy but died of his disease 5 months later.

Distant spread is more common with nasopharyngeal carcinoma than with other head and neck squamous cell carcinomas. The most frequent sites of distant metastases are bone, lung, liver, and distant lymph nodes [1,2]. A clinical diagnosis of metastatic cancer in the esophagus arising from nasopharyngeal carcinoma is extremely rare. To the best of our knowledge, this type of metastasis has not been previously described in the English literature.

In conclusion, secondary esophageal carcinoma from nasopharyngeal carcinoma is rare, presents as a distant recurrence, and has a very poor prognosis.

Endoscopy_UCTN_Code_CCL_1AB_2AC_3AB

\section{Competing interests: None}

\section{S.-Y. Yang, C.-Y. Chien}

Department of Otolaryngology, Kaohsiung Chang Gung Memorial Hospital and Chang Gung University College of Medicine,

Kaohsiung, Taiwan 


\section{References}

1 Altun M, Fandi A, Dupuis O et al. Undifferentiated nasopharyngeal cancer (UCNT): current diagnostic and therapeutic aspects. Int J Radiat Oncol Biol Phys 1995; 32: 859-877

2 Hsu MM, Tu SM. Nasopharyngeal carcinoma in Taiwan. Clinical manifestations and results of therapy. Cancer 1983; 52: 362-368
Bibliography

DOI http://dx.doi.org/

10.1055/s-0032-1326274

Endoscopy 2013; 45: E120-E121

(c) Georg Thieme Verlag KG

Stuttgart · New York

ISSN 0013-726X
Corresponding author

\section{C.-Y. Chien, MD}

Department of Otolaryngology Kaohsiung Chang Gung Memorial Hospital 123 Ta-Pei Road

Niao-Song District

Kaohsiung 833

Taiwan

Fax: +886-7-7313855

cychien3965@adm.cgmh.org.tw 